\title{
EFECTO DE LA MADURACIÓN, COCIÓN Y CONGELAMIENTO SOBRE LA SUAVIDAD, RENDIMIENTO Y CARGA MICROBIANA DEL CORTE DE SOLOMO (OUTSIDE) ${ }^{1}$
}

\author{
Alejandro Chacón Villalobos ${ }^{2}$
}

\begin{abstract}
RESUMEN
Efecto de la maduración, cocción y congelamiento sobre la suavidad, rendimiento y carga microbiana del corte de solomo (outside). Se utilizaron 115 solomos (outside según sistema de corte americano) con peso medio de $2,9 \mathrm{~kg}$. Cinco fueron destinados a pruebas de variación interna en fuerza de corte y 10 para análisis químicos de composición preliminares. Ochenta fueron empacados al vacío y madurados a $7{ }^{\circ} \mathrm{C}$ durante siete días y posteriormente divididos en cuatro subgrupos de 20 unidades. Un subgrupo se congeló a $-25^{\circ} \mathrm{C}$ durante un mes. El segundo y tercero se cocinaron en una marmita hasta alcanzar una temperatura interna estimada de $70{ }^{\circ} \mathrm{C}$. Al terminar la cocción el tercer subgrupo se congeló como se describió antes. El cuarto solo se maduró (siete días $/ 7{ }^{\circ} \mathrm{C}$ ). Veinte piezas frescas constituyeron el grupo control. Se determinó la fuerza de corte para las 100 piezas tratadas empleando el método de la American Meat Science Association. Se estimó la calidad microbiológica y las pérdidas de peso de cada tratamiento. El pH de las piezas frescas, y de aquellas sometidas a los procesos de maduración fueron determinados. El solomo fresco mostró una alta variabilidad en la fuerza de corte, estimada en $7,45 \mathrm{~kg} / \mathrm{cm}^{2}$, y con una composición ligeramente alta en tejido conectivo $(2,9 \%)$. Presentó además valores de $\mathrm{pH}$ bajos que no se ven significativamente incrementados por la maduración. Todos los tratamientos aumentaron en algún grado la suavidad del solomo y presentaron recuentos microbianos sanitariamente aceptables. La menor mejoría en la suavidad correspondió a aquellos tratamientos que involucraron la cocción y la congelación. El tratamiento de maduración durante 7 días a $7{ }^{\circ} \mathrm{C}$ fue el que generó mejores resultados generales.
\end{abstract}

Palabras clave: Solomo, carne, suavidad, maduración, cocción, congelación.

\begin{abstract}
Effect of aging, cooking and freezing on tenderness, yield and microbial counts of "solomo" (outside) meat cuts. A total of 115 outside cuts with a medium weight of $2.9 \mathrm{~kg}$ were used. Five samples were used for the estimation of the internal variation of the cut force and 10 samples for chemical composition evaluation. Eighty cuts were vacuum-packed and then aged for seven days at $7{ }^{\circ} \mathrm{C}$. After aging, four subgroups of 20 units were established. The first was frozen at $-25^{\circ} \mathrm{C}$ for a month. The second and the third subgroups were cooked by immersion in hot water until the internal temperature reached $70{ }^{\circ} \mathrm{C}$. At the end of the cooking process, the third group was frozen as described above. The fourth group only underwent aging (seven days $/ 7^{\circ} \mathrm{C}$ ). Twenty fresh cuts were used as a control group. The "cut force" of the 100 treated cuts was evaluated by the methods of the American Meat Science Association. Microbiological quality and weight losses of every treatment, and the $\mathrm{pH}$ values for fresh and aged cuts were evaluated. Fresh cuts showed high variability in tenderness, expressed as a function of the cut force estimated in $7.45 \mathrm{~kg} / \mathrm{cm}^{2}$, and exhibited slightly higher proportion of connective tissue $(2.9 \%)$. Outside fractions, both fresh and aged, showed low $\mathrm{pH}$ values, but did not show statistically significant differences among each other. Microbiological evaluations showed that all treatments were sanitarily executed. All treatments improved outside tenderness. The treatments composed by cooking and by freezing showed the least improvement. The best results were obtained under aging during seven days at a temperature of $7{ }^{\circ} \mathrm{C}$.
\end{abstract}

Key words: Ouside, meat, tenderness, aging, cooking, freezing.

\section{INTRODUCCIÓN}

Aunque los gustos de muchos de los consumidores alrededor del mundo se han modificado a causa del desarrollo y del ritmo de vida, una expectativa siempre se ha mantenido constante: la carne debe ser suave (Woodhouse 1998). La razón anterior justifica el hecho de que la suavidad sea reconocida, por una gran cantidad

\footnotetext{
1 Recibido: 27 de enero, 2005. Aceptado: 30 de agosto, 2005.

2 Estación Experimental Alfredo Volio Mata. Facultad de Ciencias Agroalimentarias. Universidad de Costa Rica. Correo electrónico: achaconv@cariari.ucr.ac.cr
} 
de investigadores, como el parámetro más importante con que el consumidor juzga la calidad de la carne (Joseph 1971; Stouffer 1975; Polidori et al. 1996). Algunos autores incluso consideran que la suavidad es crucial para la aceptación de la carne en el ámbito mundial (Menzies 1997).

A diferencia de muchos parámetros de calidad como el sabor, el color y el contenido de grasa, la suavidad de una pieza es siempre la más variable y menos evidente de las características ligadas a la calidad (Woodhouse 1998). Es por ello que estudiar el problema de la inconsistencia en la suavidad de la carne, y de los diferentes procesos relacionados con su mejoramiento, se considera actualmente como la mayor preocupación en la industria (Koohmaraie et al. 1996). En consecuencia, existe un gran potencial económico para aquellos procesos que puedan generar, bajo bases constantes y controladas, carne de una suavidad superior (Woodhouse 1998), sobre todo cuando por lo menos el $25 \%$ de las piezas evaluadas a escala mundial son consideradas excesivamente duras (Olson 1998).

El descubrimiento de que la contracción muscular es una de las principales causas de la dureza en la carne, ha permitido concluir, que las operaciones industriales ante y post-mortem adecuadamente aplicadas pueden ser más influyentes en la dureza final que los factores presentes en el animal vivo (Polidori et al. 1996). Entre tales factores se puede citar el estrés, el tratamiento previo antes de la matanza y el contenido de grasa (Joseph 1971). El colágeno total, cuya cantidad depende de la edad, sexo y raza, es generalmente aceptado como poseedor de un papel significativo más no preponderante en la dureza final, aún a pesar de que no se ha demostrado una correlación directa entre ambos factores (Bailey 1979).

Basándose en la dureza como una función del grado de contracción muscular y del contenido de colágeno, muchos han sido los métodos sugeridos para el mejoramiento de la suavidad. Se ha estudiado entre ellos el estiramiento mecánico (Mueller 1990), el uso de extractos enzimáticos de frutas (Quaglia et al. 1992) o de origen bacteriano (Takagi et al. 1992), la estimulación eléctrica (Bostan et al. 2001; Hwang et al. 2003) y las altas presiones (Schilling et al. 2002). Todos ellos buscan causar un alargamiento del sarcómero o una ruptura de la integridad de las fibras musculares o del tejido conectivo (Savell 1998), objetivos que pueden ser alcanzados ya sea por daño estructural, o por un aceleramiento metabólico observable en el almacenamiento durante la maduración de la pieza (Bruce et al. 1990).

Datos obtenidos en investigaciones recientes, sugieren que la proteólisis post-mortem de las proteínas miofibrilares de la carne sufridas en el almacenamiento durante la maduración de la pieza, es la principal causa del mejoramiento de la suavidad (Koohmaraie 1994; Doumit et al. 1999), y enfatizan sustancialmente la importancia de la degradación protéica como factor clave en la suavidad final (Buts et al. 1987).

Amplia evidencia experimental indica que la ruptura de las proteínas claves responsables de la estructura muscular observada en el almacenamiento durante la maduración, está asociada a un sistema enzimático (Taylor et al. 1995). Dicho sistema es el de las denominadas proteasas neutrales dependientes del calcio o Calpaínas integrado por las enzimas $\mu$-calpaína, $\mu / \mathrm{m}$-calpaína y mcalpaína además del inhibidor calpastatina (Geesink et al. 1998; Pringle et al. 1996). Estas enzimas constituyen un sistema endógeno altamente dependiente del $\mathrm{Ca}^{2+}$ que se encuentra localizado en el citosol (Pringle et al. 1995). Su forma de acción radica en la ruptura gradual de la línea $\mathrm{z}$ al liberar la $\alpha$-actinina que une los delgados filamentos en esa zona (University of Guelph, 1998). Los efectos descritos con respecto a la acción de estas enzimas evidencian el papel primario que cumplen en el aumento de la suavidad post-mortem registrado en el almacenamiento durante el cual se experimenta la maduración (Dobbie et al. 1997), a lo que se suman estudios que demuestran que más del $90 \%$ del incremento en la suavidad que ocurre durante el almacenamiento puede ser atribuido a la acción de este sistema enzimático (Alayan 1997). Métodos de suavización por estimulación de las calpaínas con infusiones de $\mathrm{CaCl}_{2}$ son ampliamente discutidos por la literatura (Wheeler et al. 1992; Wheeler et al. 1997).

Adicionalmente a la maduración, procedimientos industriales simples o combinados como el empacado al vacío, la cocción y congelación pueden tener un efecto importante en la suavidad. Es de interés evaluar primariamente el potencial de estas operaciones sencillas como fuente prístina de mejoramiento para la suavidad de la carne, no solo por los costos de implementación de muchos de los tratamientos antes descritos, si no también por los problemas de espacio, tiempo, contaminación e incluso de confiabilidad que pueden derivar de los mismos, especialmente en sistemas de producción a pequeña escala (Maltin et al. 2003; Chacón 2004).

El empacado al vacío ha demostrado favorecer ampliamente la suavidad de la carne y mejorar la vida útil de piezas empacadas de este modo (Heinemann y Pinto 2003; Huerta-Leidenz et al. 2004; Vázquez et al. 2004). El empaque favorece, además, la aceptabilidad del producto final por parte del consumidor (Costell 1992). Por medio del proceso de cocción, eventualmente se favorecería más la suavidad de la pieza por desnaturalización y gelatinización del tejido conectivo. 
El proceso de congelación, a su vez, se justifica no sólo por el hecho de que éste ha demostrado tener un potencial para mejorar la suavidad de la carne (Whipple et al. 1992), si no que, además, este proceso corresponde a la mejor acción preventiva ante la aparición de organismos patógenos que podría darse en el producto cocinado al vacío, si este es mantenido a temperaturas por encima de $0{ }^{\circ} \mathrm{C}$ (Jeremiah 1998). Como parámetros de calidad sanitaria es factible evaluar, al ejecutar estas operaciones simples antes descritas y en forma básica y preliminar, el recuento de bacterias lácticas, de coliformes y la presencia o ausencia de esporulados anaerobios mesófilos. Las determinaciones anteriores se justifican por la importancia de dichos microorganismos en productos cárnicos empacados y cocinados al vacío (Ahn y Stiles 1990; Vanderzant et al. 1992; Nicolai et al. 1993).

En Costa Rica, el corte de carne conocido como solomo (outside) es tradicionalmente señalado como el de mayor dureza. Esta condición limita el valor económico y las posibilidades de procesamiento (Solís 1999). Su textura, así como los efectos del empacado al vacío, la maduración, la cocción y el congelamiento no han sido claramente estudiados en el pasado, factor que limita la posible adopción de medidas técnicas y económicas, basadas en la dotación técnica particular ya instalada que estén orientadas al aumento de la terneza de este producto. Estudios en este sentido, permiten una caracterización de la variación e interacción de estos parámetros sobre la variable respuesta de la fuerza de corte, generando un mejor entendimiento de las posibilidades tecnológicas de este corte de carne.

Se presenta este estudio con el objetivo de evaluar el efecto individual y combinado de los procesos de maduración, cocción y congelamiento sobre el rendimiento, la carga microbiana y la suavidad final del corte de solomo bovino.

\section{MATERIALES Y MÉTODOS}

\section{Localización y materia prima}

Los procedimientos de maduración, empacado, cocción y congelamiento así como los análisis químicos y los recuentos microbiológicos se realizaron en el Laboratorio de Control de Calidad de Coopemontecillos RL, ubicado en la provincia de Alajuela. La determinación de la fuerza de corte se llevó a cabo en el Laboratorio de Análisis Sensorial del Centro Nacional en Ciencia y Tecnología de Alimentos (CITA), de la Universidad de Costa Rica, ubicado en San Pedro de Montes de Oca.
Se utilizaron 115 cabezas de ganado cebuino (machos), con una edad aproximada de 3,5 años. Esta raza representa la mayor parte del ganado sacrificado en Coopemontecillos R.L (Solís 1999). Las muestras fueron piezas de solomo recolectadas por medio de un muestreo simple aleatorio 24 horas después del sacrificio (recién finalizado el período de rigor mortis y antes de que los cambios degradativos asociados a la maduración inicien). Pasado este tiempo las canales alcanzan una temperatura de $7{ }^{\circ} \mathrm{C}$ en las cámaras de refrigerado y puede ejecutarse un deshuesado sanitariamente adecuado. Las piezas se obtiene de la media canal tal y como muestra la Figura 1. De cada cabeza de ganado se obtuvo una pieza de solomo. Del total de piezas obtenidas se destinaron cinco para una evaluación preliminar de la variación interna de la fuerza de corte, 10 para las evaluaciones de composición química y 100 para el estudio de los tratamientos aplicados. Pruebas preliminares definieron el peso medio de los solomos en 2,9 $\pm 0,1 \mathrm{~kg}$.

El solomo se encuentra ubicado en la zona de los cuartos traseros del animal, y durante el deshuese se obtiene preliminarmente en la forma de un corte en bruto que posteriormente es segmentado eliminándose la punta, que corresponde a otro corte llamado "punta de lomo", y la "cola" que no forma tampoco parte del solomo como tal. Lo mismo sucede con una pequeña sección lateral de poco valor. Al final se obtiene en todos los casos una pieza central muy uniforme en forma de paralelepípedo (Figura 1).

El solomo fue escogido con fines experimentales dada la baja demanda comercial derivada de su alta dureza y por el interés existente en mejorar su aceptación.

\section{Evaluación preliminar de la variación en la fuerza de corte a lo largo de piezas de solomo fresco}

Preliminarmente, se evaluó la fuerza de corte a cinco piezas de solomo fresco recolectadas empleando un muestreo simple aleatorio.

Cada una de las piezas fue seccionada transversalmente en seis filetes consecutivos de 2,54 cm de ancho. Cada filete representó una posición determinada a lo largo del cuerpo de cada solomo partiendo de la posición más cercana a la punta y de ahí hacia la cola. Gracias a la uniformidad en cuanto a forma y tamaño de los solomos, se pudo comparar filetes de diferentes piezas que tienen aproximadamente una misma posición dentro de cada una de ellas.

A cada uno de los seis filetes provenientes de cada uno de los solomos (30 en total), debidamente identificados según su ubicación, le fue determinada la fuerza 


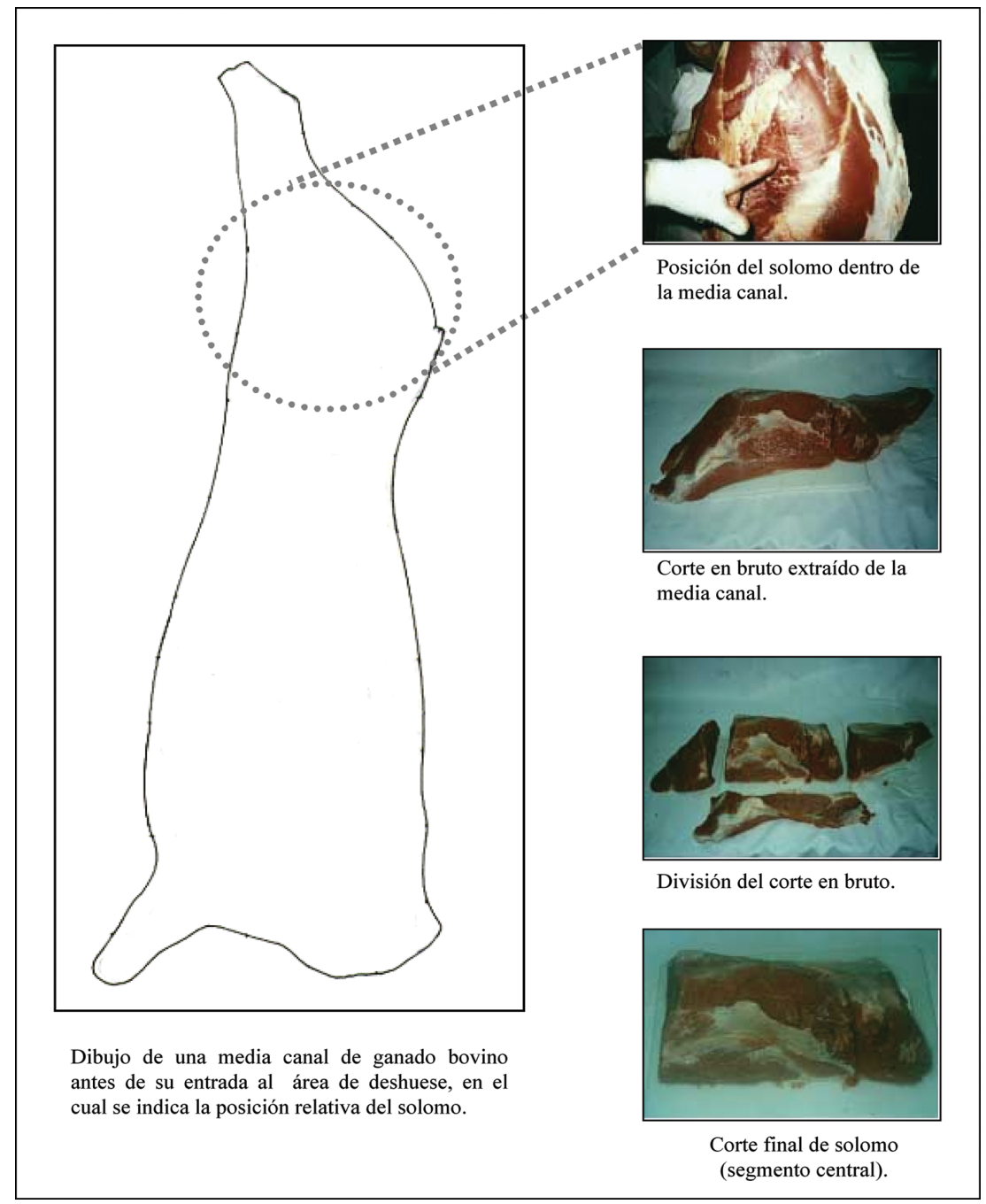

Figura 1. Esquema ilustrativo de la obtención de un corte de solomo a partir de una media canal sometida al deshuesado. San José, Costa Rica. 2005.

de corte expresada como $\mathrm{kg} / \mathrm{cm}^{2}$ en nueve diferentes posiciones internas aleatorias y con las fibras musculares perpendiculares a la dirección de corte, empleando un texturómetro Instron modelo 1.000 funcionando en el modo "Peak", para una celda de $50 \mathrm{~kg}$, un rango de $20 \mathrm{~kg}$ y una velocidad de $50 \mathrm{~mm}$ por minuto, y según los procedimientos de la American Meat Science Association (American Meat Science Association 1978). En total se efectuaron 270 mediciones para todos los solomos de las cuales 20 fueron desechadas debido a la presencia de cartílagos y otros cuerpos extraños tal y como indica la American Meat Science Association.

A partir de los datos obtenidos se efectuó un análisis de varianza para estudiar la variabilidad de la fuerza de corte, y determinar así diferencias significativas de una posición a otra dentro de los solomos. Posteriormente, se efectuó una regresión lineal para observar la dureza media del corte según su posición y estudiar si existe un modelo estadístico satisfactorio que explique como la dureza del solomo varia significativamente en función de la posición de donde se tomó el filete.

Empleando los valores de fuerza de corte para todos los filetes de las cinco piezas, se calculó la desviación estándar. Con base en este resultado y según las recomendaciones de González (1999)³ ${ }^{3}$, se estimó estadísticamente un tamaño de muestra de 20 solomos para cada uno de los tratamientos y el grupo de control, al efectuar las determinaciones de fuerza de corte posteriores.

\footnotetext{
3 GONZÁLEZ, M. 1999. Técnicas de evaluación estadística. Escuela de Estadística de la UCR. San Pedro de Montes de OCa, San José, Costa Rica. Entrevista.
} 


\section{Composición química}

Se efectuó un análisis químico a 10 piezas de solomo frescas, recolectadas empleando un muestreo simple aleatorio, homogenizándolas adecuadamente de manera que la muestra fuese representativa, y conservada adecuadamente. El análisis involucró:

a) Determinación del porcentaje de humedad por el método de secado rápido con microondas, AOAC 985.14 (A.O.A.C. 2000).

b) Determinación del contenido de grasa, AOAC 960.39 (A.O.A.C. 2000).

c) Separación y cuantificación de las proteínas del tejido muscular por medio del método propuesto por Herrera (1997): Este procedimiento involucra una separación con buffers de fosfatos de diferente concentración salina, y posterior determinación espectrofotométrica por el método de Biuret de las proteínas sarcoplásmicas y miofibrilares, así como la determinación gravimétrica de las proteínas del colágeno y demás tejido conectivo (proteínas estromales).

Los resultados para cada prueba se evaluaron calculando intervalos de confianza del 99\% (Haber y Runyon 1973). Se conservó para el dato final la mayor incertidumbre obtenida.

\section{Tratamientos aplicados al solomo}

Se efectuaron en total cuatro tratamientos empleando en cada uno de ellos 20 piezas de solomo cuya masa se determinó en el momento mismo del muestreo. Los tratamientos fueron: maduración; maduración y posterior congelación; maduración de los solomos y ulterior cocción, y finalmente maduración, cocción y posterior congelación. También se estableció un grupo control de 20 piezas frescas sin tratamiento.

La maduración es común a todos los tratamientos no sólo por ser un proceso estándar en la industria de los cárnicos, si no por el interés en caracterizar la acción enzimática teóricamente esperada sobre la dureza. En términos generales, en la industria la congelación y la cocción se aplican con posterioridad a la maduración. Además, la cocción como procedimiento puede destruir irreparablemente las enzimas involucradas en los cambios estructurales madurativos que originan mucha de la suavidad de la carne, y suele generar mucha pérdida de fluidos en carnes muy frescas. Es por ello que se consideró como lo más prudente estudiarla como un proceso posterior a la maduración.
Los cuatro tratamientos estuvieron compuestos de manera individual o combinada por tres procedimientos básicos:

\section{Procedimiento de maduración}

Se tomaron las 80 piezas en bruto de solomo fresco a ser sometidas a los cuatro tratamientos directamente de la línea de deshuese, se prepararon tal y como indica la Figura 1, se pesaron, y se empacaron individualmente al vacío en una bolsa para empaque y cocción CRYOVAC CN-510. Este empaque posee una estructura multicapas de muy alta resistencia y excelentes características de barrera que evitan sustancialmente las pérdidas de humedad. Una vez empacadas todas las piezas, se almacenaron en una cámara de refrigeración que mantuvo una temperatura de $7{ }^{\circ} \mathrm{C}$ durante un período de siete días. Se permitió dentro de los anaqueles una distancia entre piezas de $2,5 \mathrm{~cm}$ en la dimensión horizontal y de $10 \mathrm{~cm}$ en la dimensión vertical con la finalidad de permitir una adecuada circulación del aire.

\section{Procedimiento de cocción}

Se emplearon cuarenta de las ochenta piezas totales que ya habían pasado por el procedimiento previo de maduración. Estas se cocinaron empacadas al vacío en el mismo empaque en que fueron maduradas (CRYOVAC CN-510) hasta lograr una temperatura interna estimada en el centro de $70{ }^{\circ} \mathrm{C}$. El proceso de cocción correspondió a un proceso escalonado en tres tiempos donde la pieza se cocinó en una marmita con agua a 65 ${ }^{\circ} \mathrm{C}$ durante la primera hora, a $70{ }^{\circ} \mathrm{C}$ durante la segunda hora y finalmente a $75^{\circ} \mathrm{C}$ hasta obtener la temperatura interna estimada de $70{ }^{\circ} \mathrm{C}$. La práctica industrial recomienda este tipo de procedimientos para aquellas piezas duras y con contenidos de colágeno importantes (Solís 1999) ${ }^{4}$. Para estimar la temperatura interna final, dos piezas de solomo adicionales se incorporaron dentro de la marmita con el único propósito de insertar en ellas un termopar que midió su temperatura central a lo largo del proceso. Al finalizar la cocción estas piezas adicionales se descartaron.

\section{Procedimiento de congelación}

Para este procedimiento se utilizaron 40 solomos que correspondieron a un grupo de 20 solomos que

\footnotetext{
4 SOLÍS, E. 1999. Suavización por inyección de cloruro de calcio y discusión de métodos de análisis implementados en el laboratorio de control de calidad de Coopemontecillos R.L. Alajuela, Costa Rica. Entrevista.
} 
habían pasado antes por el proceso de maduración, y a un segundo grupo de 20 solomos que habían sufrido maduración y posterior cocción. Los solomos, aún dentro del empaque original, se almacenaron en una cámara de congelación a una temperatura de $-25{ }^{\circ} \mathrm{C}$ donde se mantuvieron durante un período de 30 días.

Finalizado el tiempo de almacenamiento, las piezas se trasladaron a una cámara de refrigeración a $7{ }^{\circ} \mathrm{C}$ dónde se descongelaron durante las 18 horas anteriores a las determinaciones.

\section{Determinaciones previas a la medición de la fuerza de corte}

Finalizados los procedimientos de maduración, cocción y congelado aplicados a los 80 solomos en sus diferentes combinaciones, se efectuaron las siguientes determinaciones:

Determinación de la pérdida de peso durante los tratamientos

Se seleccionaron al azar 15 piezas empacadas de las 20 resultantes de cada tratamiento, y se procedió a abrir su empaque, drenando cualquier líquido en su interior. Empleando los datos de masa inicial y los valores de masa final después de drenar, se calculó el porcentaje de pérdida en cada pieza para un intervalo de confianza de 99,9\% (Haber y Runyon 1973).

Determinación del pH previo y posterior a la maduración

La determinación de pH se efectuó para las piezas que recibieron maduración como único tratamiento, así como en las del grupo control.

De las 15 piezas correspondientes al tratamiento de maduración, que fueron drenadas durante la determinación de las pérdidas de peso, se escogieron al azar 10 y se procedió a extraer una muestra representativa homogénea destinada a medir el $\mathrm{pH}$. Se hizo lo mismo con 10 solomos frescos del grupo de control. Una vez obtenidos los datos de $\mathrm{pH}$, se efectuó un análisis de varianza con los datos para determinar si existía una diferencia significativa entre las piezas maduradas y el grupo control. Se calculó el intervalo de confianza para un $99,9 \%$ de estas mediciones en el tratamiento y el grupo control (Haber y Runyon 1973).

\section{Determinaciones microbiológicas}

En todos los tratamientos, se efectuaron análisis microbiológicos empleando como fuente de la muestra las cinco piezas por tratamiento que no fueron drenadas para determinar las pérdidas de peso. Además se emplearon cinco piezas de solomo fresco del grupo control. Se procedió de esta manera para asegurar que los recuentos reflejaran la posible contaminación durante los tratamientos, pero no la posible recontaminación debida a la manipulación asociada al drenado y al pesaje. Los análisis microbiológicos efectuados fueron recuento total aerobio, determinación de bacterias lácticas, determinación de esporulados anaerobios mesófilos y determinación de coliformes, todos según los procedimientos enunciados por Vanderzant y Splittstoesser (1992).

El recuento total de microorganismos aerobios se efectuó únicamente para el grupo control a fin de tener una estimación de la carga aerobia inicial.

\section{Determinación de la fuerza de corte}

Determinadas las pérdidas de peso, las estimaciones de $\mathrm{pH}$ y las evaluaciones microbiológicas, se procedió a obtener un corte transversal de $2,54 \mathrm{~cm}$ de grosor (filete), efectuado en el sector central de cada una de las 20 piezas de solomo que integraron los cuatro tratamientos y el grupo control.

Los filetes obtenidos se empacaron al vacío en bolsas individuales para facilitar su transporte, y se depositaron en un bolso térmico a una temperatura de refrigeración durante el breve período del traslado (1 hora).

$\mathrm{Al}$ arribar al laboratorio, aquellos filetes que como parte del tratamiento fueron sometidos a un procedimiento de cocción, se encontraban ya listos para la medición de la fuerza de corte. Los que arribaron crudos, se cocinaron a la parrilla en un horno eléctrico a $300{ }^{\circ} \mathrm{C}$ en modo "Broil", de acuerdo al método descrito por AMSA (1978), hasta que la temperatura interna llegó a los $55^{\circ} \mathrm{C}$, luego se voltearon, y cuando alcanzaron una temperatura interna de $70{ }^{\circ} \mathrm{C}$ se sacaron del horno. Para la medición de las temperaturas se utilizó un termopar insertado en el centro de cada filete, el cual se encontraba conectado a un aparato termo registrador Cole-Palmer. Finalizada la cocción se permitió un período de dos horas para el enfriamiento. Posteriormente se eliminaron los bordes de cada uno de los filetes y se extrajo aleatoriamente de cada uno de ellos un mínimo 
de cinco muestras cilíndricas de 1,27 cm de diámetro y $2,54 \mathrm{~cm}$ de largo, procurándose que cada una de ellas no presentará irregularidades como nervios, venas o cartílagos. Dichas muestras se obtienen utilizando una herramienta hueca cilíndrica afilada que es parte del texturómetro utilizado, y con la que se efectúa el corte de los filetes. Se determinó a continuación la fuerza de corte en $\mathrm{kg} / \mathrm{cm}^{2}$ de cada cilindro empleando para ello un texturómetro Instron modelo 1000 funcionando en el modo "Peak", para una celda de $50 \mathrm{~kg}$, un rango de 20 $\mathrm{kg}$ y una velocidad de $50 \mathrm{~mm}$ por minuto.

\section{Análisis estadístico de los datos de fuerza de corte}

A partir de los promedios obtenidos para cada uno de los tratamientos y para el grupo de control, se efectuó primeramente un análisis de varianza tomando la fuerza de corte como variable dependiente para verificar la existencia de evidencia estadística que demuestre o rechace la hipótesis nula de que los promedios de cada tratamiento no son significativamente diferentes entre sí. Una vez demostrado lo anterior, se llevó a cabo una prueba Post Hoc de Scheffé de comparación múltiple de promedios para la fuerza como variable dependiente, que es una metodología de fácil interpretación, está estandarizada para cantidades desiguales de datos y es de las más conservadoras. Esto último es útil en casos donde la variabilidad es muy acentuada (Williams 1959; Scheffé 1959). Este procedimiento ofrece muchas ventajas para los propósitos de esta investigación, como el garantizar que el error experimental tipo I nunca excederá la condición $\mathrm{p} \leq 0,05$ sin importar la cantidad de comparaciones a efectuar. En la prueba, cada promedio por tratamiento se comparó estadísticamente con cada uno de los promedios de los demás tratamientos en busca de diferencias significativas. La prueba de Scheffé, agrupó a los tratamientos en grupos homogéneos significativamente diferentes de los demás según la fuerza de corte promedio, es decir, en grupos dentro de los cuales no existen diferencias significativas. De esta forma, si los promedios para la fuerza de corte de dos o más tratamientos quedan ubicados dentro de un mismo grupo, esto significa que los efectos de esos tratamientos en la fuerza de corte no son significativamente diferentes entre sí.

En el procesamiento de los datos de fuerza de corte, así como en todas las evaluaciones estadísticas realizadas en este estudio, se empleó el programa estadístico SPSS versión 8.0 para ambiente Windows. En todas las determinaciones las potencias de prueba fueron iguales o superiores al $90 \%$.

\section{RESULTADOS Y DISCUSIÓN}

\section{Evaluación preliminar de la fuerza de corte a lo lar- go de cinco piezas de solomo fresco}

A partir de los resultados del análisis de varianza se obtuvo evidencia estadística suficiente $(\mathrm{p} \leq 0,001)$ para afirmar que existen diferencias significativas entre los promedios de cada filete, lo que quiere decir que la suavidad de las diferentes posiciones de corte dentro de los solomos frescos no fue uniforme. Una prueba de Tuckey posterior $(\alpha=0,05)$ señaló diferencias significativas solo para las posiciones 1, 2 (borde) entre sí pero no entre estas y las demás. Se aplicó una regresión lineal para la variable posición con la finalidad de explicar la variación existente.

En la Figura 2, puede observarse la alta dispersión de la fuerza de corte medida en cada una de las posiciones en los cinco solomos.

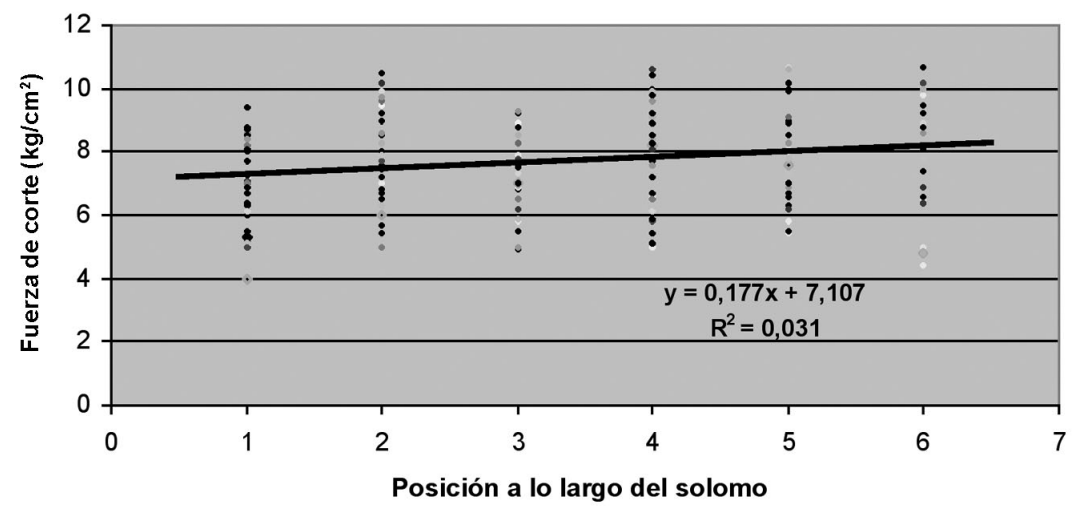

Figura 2. Fuerzas de corte estimadas en cada una de las posiciones internas de los solomos de control empleados en la prueba preliminar. San José, Costa Rica. 2005. 
La regresión lineal arrojó una significancia de 0,006, lo cual indica una tendencia lineal creciente para la fuerza de corte de "cabeza" a "cola". No obstante, el porcentaje de varianza explicada por el modelo lineal es de tan solo 3,1\% (coeficiente de correlación lineal de 0,031 ) siendo la tendencia lineal poco perceptible y distorsionada por la alta variabilidad entre solomos, lo cual no puede ser utilizado para predecir.

Con base en lo anterior, se decidió tomar en adelante un filete ubicado en la zona central del solomo en un intento de estandarizar las pruebas experimentales posteriores, dado que con un modelo tan pobre estadísticamente daba lo mismo tomar la muestra en cualquier posición dentro del solomo.

La desviación estándar para las mediciones fue de 2,54. Con base a este resultado se estimó un tamaño de muestra de 20 solomos por tratamiento para las determinaciones de fuerza de corte posteriores.

\section{Composición química}

Los porcentajes para los componentes evaluados se presentan con el mayor valor de incertidumbre calculada en el Cuadro 1.

Cuadro 1. Composición química porcentual estimada para el solomo. San José, Costa Rica. 2005.

\begin{tabular}{lc}
\hline \multicolumn{1}{c}{ Componente } & Porcentaje $(\mathbf{m} / \mathbf{m})^{(\mathbf{1})}$ \\
\hline Humedad & $75 \pm 1 \%$ \\
Grasa & $2,3 \pm 0,3 \%$ \\
Proteínas: & \\
$\quad$ Sarcoplasmáticas & $5,1 \pm 0,4 \%$ \\
$\quad$ Miofribrilares & $10 \pm 1 \%$ \\
$\quad$ Colágeno (Proteínas estromales) & $2,9 \pm 0,3 \%$ \\
\hline
\end{tabular}

(1) Porcentaje masa en masa calculado sobre el peso húmedo de la muestra.

El análisis proximal efectuado fue parcial por cuanto no se determinaron carbohidratos, vitaminas y otras sustancias misceláneas no proteicas. Se limitó el análisis a aquellos componentes que fueron considerados de importancia con relación a la suavidad.

En la Figura 3, se comparan los resultados obtenidos contra los datos porcentuales citados por Cole y Lawrie (1975), que corresponden a la composición típica del músculo después del rigor mortis pero antes de

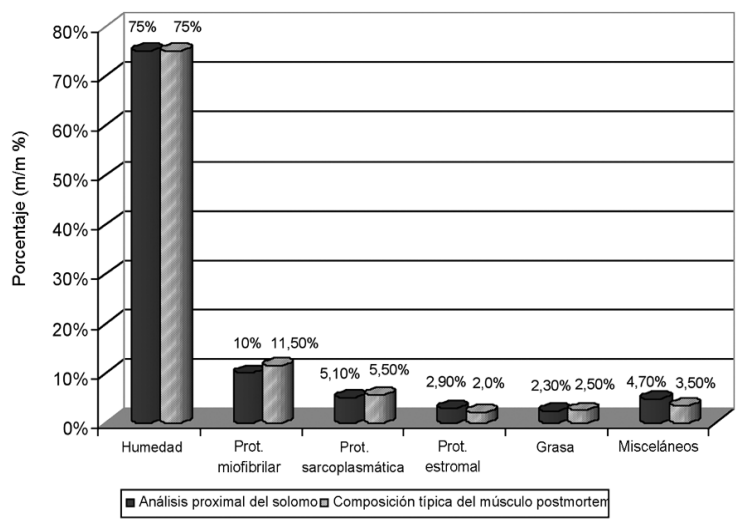

Figura 3. Comparación del análisis proximal del solomo fresco control y los valores de composición porcentual típicos del músculo de mamíferos adultos después de la resolución del rigor mortis. San José, Costa Rica. 2005.

los cambios degradativos post mortem, es decir antes del proceso de maduración. Puede verse claramente que los valores porcentuales obtenidos al analizar las piezas de solomo son muy similares a los correspondientes a un músculo típico con posterioridad a la resolución del rigor mortis. No se da ningún comportamiento que haga sospechar que la dureza del solomo pueda estar vinculada a una anormalidad acentuada en cuanto a composición se refiere. Es notorio que el contenido de proteína miofibrilar es comparativamente menor, mientras que el contenido de proteínas del estroma y del tejido conectivo (colágeno y elastina) es mayor, aún si se toma en cuenta la incertidumbre calculada para el porcentaje de estos componentes. Según Savell (1998), es posible esperar una reducción del contenido de proteína miofibrilar al haber una mayor cantidad de proteína estromal presente, condición que al parecer podría estarse corroborando en este caso.

Los resultados evidencian que el tejido conectivo es un constituyente importante del solomo, siendo posible que sea un factor de suficiente peso para explicar parte de su dureza, sobretodo si se considera que al ser los animales machos y de 3,5 años de edad, la magnitud de los enlaces cruzados entre las moléculas de tropocolágeno que forman dicho tejido puede ser muy significativa.

El contenido de grasa determinado $(2,4 \%)$, no es lo suficientemente elevado como para tener un efecto mejorador sobre la suavidad, y es de hecho más característico de cortes duros que de cortes suaves, donde la grasa alcanza porcentajes cercanos a un $8 \%$ (Texas A\&M University 1999). No se da un efecto de marmoleo o "marbling" apreciable. 
El porcentaje de humedad obtenido para el solomo es el típico esperado en muestras de carne fresca (Cole et al. 1975; University of Illinois Animal Sciences Department 1999). En teoría, la relación entre agua y proteína es para la carne de aproximadamente 3,7 a 1 (University of Illinois Animal Sciences Department 1999), lo cual es muy similar a la relación 4,1 a 1 registrada para el solomo. Esto confirma una vez más que la composición porcentual del solomo guarda en términos generales la tendencia típica. El hecho de que el contenido de agua obtenido sea común al de otras piezas de menor dureza que el solomo, hace que no se pueda afirmar si existe un efecto del agua en la suavidad general. De hecho este es un tema que actualmente es fuente de discusión entre los científicos (Romans $2000)^{5}$. El porcentaje de humedad tiene mayor importancia cuando se aplican métodos sensoriales para evaluar la suavidad, especialmente porque puede llegar a confundirse la jugosidad con la suavidad, lo cual no suele ser tan común en mediciones instrumentales de la fuerza de corte (Romans 2000) $)^{5}$. Por los resultados obtenidos en el análisis proximal puede especularse que el solomo tendrá una jugosidad aceptable, pero no se puede asegurar el impacto de la misma en la aceptación hasta que pruebas sensoriales posteriores se realicen.

Basándose en las diferencias composicionales encontradas, no existen argumentos que señalen que los mecanismos de contracción muscular durante el rigor mortis o la degradación propia de los cambios postmortem, puedan tener una dinámica significativamente diferente en el solomo a la descrita por la teoría para los cortes en general.

\section{Determinación de la pérdida de peso atribuida a los tratamientos}

La pérdida porcentual de peso atribuible a cada uno de los tratamientos es un criterio importante, pues su magnitud no sólo tiene implicaciones económicas, si no que además es indicador de una disminución en la capacidad de retención de agua, aspecto que tiene implicaciones sensoriales importantes. A pesar de que la mayor parte de la pérdida corresponde al agua, muchos otros componentes como nutrientes disueltos o hasta grasa y tejido conectivo licuados durante la cocción forman parte del total perdido.

En el Cuadro 2 se resumen las pérdidas porcentuales de peso determinadas en cada uno de los tratamientos experimentales con un intervalo de confianza del 99,9\%.

\footnotetext{
5 ROMANS, J. 2000. Discusión sobre el papel del porcentaje de humedad en la suavidad de la carne. University of Illinois, Illinois. Correo electrónico.
}

Cuadro 2. Pérdida porcentual de peso estimada al final de cada uno de los tratamientos experimentales. San José, Costa Rica. 2005.

\begin{tabular}{lc}
\hline \multicolumn{1}{c}{ Tratamiento } & $\begin{array}{c}\text { Porcentaje de } \\
\text { pérdida }(\%)^{(\mathbf{1})}\end{array}$ \\
\hline Maduración $(\mathrm{M})$ & $2,5 \pm 0,3$ \\
Maduración y cocción $(\mathrm{M} \Delta)$ & $22 \pm 1$ \\
Maduración y congelación $(\mathrm{MC})$ & $5,0 \pm 0,3$ \\
Maduración, cocción y congelado $(\mathrm{M} \Delta \mathrm{C})$ & $23 \pm 1$ \\
\hline
\end{tabular}

(1) Porcentaje masa en masa..

Los tratamientos que involucraron cocción son los que generan la pérdida porcentual de peso mayor y cercana a una cuarta parte del peso total; muy superior a las pérdidas registradas en los otros tratamientos. $\mathrm{Mu}-$ cha de esa pérdida corresponde presumiblemente a la mayor cantidad del agua libre (y componentes disueltos) que permaneció en la carne después de resuelto el rigor, así como de una fracción del agua inmovilizada y muy poco del agua ligada. La pérdida se da por disminución en la capacidad de retención de agua de las proteínas debido a su coagulación por efecto térmico (Belitz y Grosch 1985). Es posible que también se registre un cierto grado de contracción de las fibras musculares, efecto que no solo expulsa agua sino que podría tener implicaciones en la dureza final (Clausen y Lassen 2002), independientemente de si el efecto térmico sobre el colágeno llega a ser acentuado.

Si se toma en cuenta que las calpaínas son destruidas durante la cocción, las pérdidas de peso y humedad no tiene importancia desde el punto de vista de la continuidad del proceso proteolítico.

No obstante los porcentajes obtenidos para los procesos de cocción, están dentro de los parámetros normales de pérdida esperada, es decir entre un $20 \%$ y un $40 \%$ (Cole y Lawrie 1975). Es importante señalar que el empacado al vacío pudo disminuir en algún grado el volumen de la pérdida no sólo durante la cocción sino a lo largo de los otros procedimientos.

Al estudiar la pérdida en el tratamiento de cocción y congelación, es notorio el hecho de que no difiere ampliamente para la muestra estudiada en donde hubo cocción pero no congelación. Esto puede explicarse en el hecho de que la cocción efectivamente eliminó la gran mayoría del agua libre remanente después del rigor mortis y una parte considerable del agua inmovilizada (Belitz y Grosch 1985). Así al tomar lugar la congelación después de la cocción, la mayor parte del agua remanente correspondía a agua ligada, la cual se pierde con dificultad. 
La congelación sin previa cocción arrojó pérdidas notoriamente menores. El porcentaje de pérdida indica un proceso de congelación y descongelación bien ejecutado. Durante la congelación se registra cierta desnaturalización de las proteínas, no obstante la misma evidencia ser muy inferior a la provocada por la cocción (Kramlich et al. 1975; Jasper y Placzek 1978; Judd 1993). Esta desnaturalización, en conjunto con el daño a la microestructura de los filamentos que va de la mano con la liberación del contenido sarcoplasmático y con una consecuente alteración del ambiente electrónico de la fibra muscular, podría explicar en parte el hecho de que las pérdidas tras la congelación sean mayores a las registradas con una simple maduración donde no se registran estos fenómenos. No obstante dependiendo de las condiciones, la degradación proteica podría por otro lado aumentar la retención de agua, por lo cual no se puede afirmar el efecto exacto hasta no efectuar más determinaciones.

En términos económicos, el tratamiento de solo maduración genera una mayor masa final de producto terminado, donde la mayor parte de la pérdida puede atribuirse al proceso de desangrado que la pieza fresca sigue dentro del empaque y secundariamente a cambios post mortem inherentes. A medida que procede la maduración, la capacidad de retención de agua aumenta como consecuencia de la liberación de proteína miofibrilar como producto de la acción proteolítica (Belitz y Grosch 1985). Ello podría explicar la razón por la cual las pérdidas de peso durante la misma no son acentuadas.

\section{Determinación del pH previo y posterior a los pro- cedimientos de maduración}

Al evaluar los resultados se obtiene que los solomos frescos tomados una vez finalizado el rigor mortis presentaron un $\mathrm{pH}$ de 5,4 $\pm 0,1$, valor que es característico (Cole et al. 1975). Por su parte, solomos madurados por siete días a $7{ }^{\circ} \mathrm{C}$ también presentaron un $\mathrm{pH}$ de $5,4 \pm 0,1$.

Con los datos de cada tratamiento se efectuó un análisis de varianza, a partir del cual no se pudo establecer la existencia de una diferencia significativa entre las piezas frescas y las maduradas en la variable respuesta $\mathrm{pH}(\mathrm{p} \geq 0,05)$. Los valores estimados de $\mathrm{pH}$, a pesar de ser relativamente bajos, pueden catalogarse dentro del límite inferior de lo considerado como normal (Troy 1994).

A juzgar por la posición del solomo dentro de la anatomía bovina, tal y como se aprecia en la Figura 1, es un músculo fuertemente vinculado con el movimiento, lo cual podría explicar que el $\mathrm{pH}$ sea relativamente bajo al final del rigor mortis al ser más extensiva la producción de ácido láctico a partir del glucógeno de reserva (Bruce y Ball 1990; Marcu y Ventila 2000).

Durante la maduración suele darse un aumento del $\mathrm{pH}$ tan bajo como 0,2 unidades (Cole y Lawrie 1975) a medida que pasa el tiempo. Para el solomo, el incremento del pH en el lapso de siete días fue mínimo e imperceptible, al menos en la metodología analítica empleada.

El grado de contracción muscular puede ser acentuado dado el $\mathrm{pH}$ final post rigor de 5,4. Si se toma en cuenta el contenido de tejido conectivo en el solomo, el cual teóricamente no sufre mayor cambio durante la maduración (Stanton 1989; Takahaski 1992; Nishimura et al. 1999), la conjunción de estos dos aspectos podría explicar mucha de la dureza particular del solomo.

Las calpaínas, en ausencia de estímulo externo, tienen un $\mathrm{pH}$ óptimo de acción in vivo de entre 7,2 y 8,2 (Wheeler 2000) ${ }^{6}$. Esto no quiere decir que para valores de $\mathrm{pH}$ como los obtenidos para el solomo, estas enzimas sean inoperantes y carentes de valor como agentes mejoradores de la suavidad. A un pH bajo como el registrado, si bien la acción es menor, la misma es suficiente para permitir teóricamente una mejora apreciable en la suavidad (Wheeler 2000) 6 .

Los valores de $\mathrm{pH}$ post rigor mesurados están sobre el punto isoeléctrico del complejo actino-miosina que es de pH 4,7 a 5,0 (Cole y Lawrie 1975), por lo cual la capacidad de retención de agua de esta pieza se encuentran dentro del intervalo de 5,0 a 5,5 donde puede considerarse como baja (Belitz y Grosch 1985), aunque no es mínima ni antes ni después de la maduración.

\section{Determinaciones microbiológicas}

Los intervalos obtenidos para las determinaciones microbiológicas se resumen en el Cuadro 4.

El recuento total aerobio efectuado se hizo con la intención de tener mayor información sobre la materia prima fresca. Por los resultados obtenidos, puede afirmarse que el solomo tiene una calidad sanitaria muy aceptable antes de dar inicio a los tratamientos. En otras palabras, es presumible que las fuentes de contaminación asociadas a los procesos de matanza, desangrado, desollado y deshuese se encuentran debidamente controladas.

\footnotetext{
6 WHEELER, T. 2000. El pH y su relación con la actividad de las calpaínas. U.S. Meat Animal Research Center. Estados Unidos de América. Correo electrónico.
} 
Cuadro 4. Intervalos de población bacteriana determinados para el solomo fresco y al final de cada uno de los tratamientos experimentales. San José, Costa Rica. 2005.

\begin{tabular}{lcccc}
\hline $\begin{array}{c}\text { Tratamiento aplicado } \\
\text { al solomo }\end{array}$ & $\begin{array}{c}\text { Recuento total } \\
\text { aerobio }(\mathbf{U F C} / \mathbf{g})^{(\mathbf{1})}\end{array}$ & $\begin{array}{c}\text { Lácticas } \\
(\mathbf{U F C} / \mathbf{g})\end{array}$ & $\begin{array}{c}\text { Coliformes }^{(2)} \\
\text { Anaerobios } \\
\text { mesófilos } \\
(\mathbf{N M P} / \mathbf{g})\end{array}$ \\
\hline Control & $10^{2}$ & $<10$ & $10 \mathrm{UFC} / \mathrm{g}$ & $<3$ \\
Maduración (M) & {$\left[10^{4}-10^{5}\right]$} & $<10 \mathrm{UFC} / \mathrm{g}$ & $<3$ \\
Maduración y cocción $(\mathrm{M} \Delta)$ & $<10$ & $<3 / \mathrm{g}$ & $<3$ \\
Maduración y congelación $(\mathrm{MC})$ & $<10$ & {$[<3-9,1]$} & $<3$ \\
Maduración, cocción y congelación $(\mathrm{M} \Delta \mathrm{C})$ & {$[10-20]$} & $<3 / \mathrm{g}$ & $<3$ \\
\hline
\end{tabular}

1) Determinado únicamente para las muestras frescas donde no se empacó al vacío.

2) En términos de UFC/g para aquellos tratamientos donde no se dio la cocción ni congelación, y en términos del $\mathrm{NMP} / \mathrm{g}$ en los demás casos. Esto debido al efecto de los tratamientos anteriores sobre la flora que hacen del $\mathrm{NMP} / \mathrm{g}$ la manera más idónea de caracterización.

$\mathrm{Al}$ observar los datos puede verse como las bacterias lácticas presentan al final de la maduración una mayor presencia en comparación con las poblaciones correspondientes a los otros microorganismos evaluados. Se cumple de este modo lo teóricamente esperado para la carne en cuanto a bacterias lácticas se refiere, siendo este comportamiento del todo normal (Dickson 20007).

Como se esperaba, aquellos tratamientos que involucraron la cocción como procedimiento final, fueron los que presentaron en términos generales los recuentos más bajos en todos los casos. Esto es particularmente notorio e importante en el caso de los bajos "Números Más Probables" (NMP) de coliformes, lo que es un fuerte indicador de que se efectuó un proceso de cocción exitoso desde un punto de vista de la calidad sanitaria. En el caso de la E. coli, parte de la flora en cuestión, los conteos típicos son del orden de menos de 10/g y contrariamente a lo que sucede con la cocción, este microorganismo puede sobrevivir a los procesos de refrigeración y congelación (Mermelstein 1993). No obstante la misma no crecerá mientras la temperatura sea inferior a los $10^{\circ} \mathrm{C}\left(\right.$ Stiles $\left.1999^{8}\right)$. Este hecho puede explicar por qué el NMP determinado al final de los tratamientos que tienen a la congelación como último procedimiento, es ligeramente mayor que en los casos que involucran tratamiento térmico.

En el caso de los esporulados anaerobios, todas las determinaciones del número más probable arrojaron re-

\footnotetext{
7 DICKSON, J.S. 2000. La calidad microbiológica de la carne. Iowa State University, College of Agriculture. Alajuela, Costa Rica. Correo electrónico.

8 STILES, M. 1999. Discusión sobre la E. coli en la carne. Alajuela, Costa Rica.
}

sultados negativos, es decir por debajo de $3 / \mathrm{g}$. Esto es un resultado importante que no sólo refleja el buen manejo de los diferentes procesos, si no que además es un significativo indicador sanitario (Delazari et al. 1980; Vanderzant et al. 1992; Jeremiah 19999; Dickson $2000^{9}$ ). Para estos microorganismos las poblaciones típicas en carne fresca no empacada pueden llegar a ser de menos de $1 \times 10^{3} / \mathrm{g}$, llegando a ser en carnes cocinadas menores a 100/g (Delazari et al. 1980; Vanderzant et al. 1992; Dickson 20007). Para productos empacados al vacío puede definirse como deseable su ausencia (Jeremiah 19998).

Al hablar de bacterias lácticas, son necesarias poblaciones iguales o superiores a $1 \times 10^{8} \mathrm{UFC} / \mathrm{g}$ para que se empiecen a presentar problemas asociados con la calidad (Dickson 2000). En el caso de los esporulados anaerobios mesófilos las poblaciones típicas en carne fresca no empacada pueden llegar a ser de menos de 1 x $10^{3} / \mathrm{g}$, llegando a ser en carnes cocinadas menores a 100/g. Para productos empacados al vacío puede definirse como deseable su ausencia.

El pH final estimado para el solomo antes y después de la maduración, difícilmente tendrá efecto inhibitorio alguno sobre las poblaciones bacterianas, que como las aquí evaluadas, crecen en la carne. Basta con comparar los valores de $\mathrm{pH}$ obtenidos (cercanos a 5,4), con aquellos a los que puede haber crecimiento de los microorganismos estudiados. La E. coli tiene un ámbito de entre 4,4 y 9,0 , las bacterias lácticas uno que va de 3,8 a 7,2 y finalmente los esporulados pueden crecer

\footnotetext{
9 JEREMIAH, L.E. 1999. Consideraciones microbiológicas sobre la cocción al vacío. L. E. Jeremiah and Associates. Alajuela, Costa Rica. Correo electrónico.
} 
para valores de $\mathrm{pH}$ superiores a 4,8 (Delazari et al. 1980; Vanderzant y Spittstoesser 1992).

Sobre la base de los recuentos obtenidos no es probable afirmar que la suavidad de la carne esté necesariamente influenciada por algún tipo de actividad bacteriana importante. No obstante estudios futuros deberán establecer si los recuentos totales de aerobios registrados de hasta $10^{5} \mathrm{UFC} / \mathrm{g}$ contienen la suficiente carga bacteriana proteolítica como para que esta pueda ejercer un efecto apreciable en la terneza de la carne. Si es posible afirmar por otra parte que el producto resultante al final de cada uno de los tratamientos experimentales es aceptable para efectos de calidad, por lo cual los procedimientos son inocuos.

\section{Evaluación de la fuerza de corte}

Un análisis de varianza preliminar para la variable fuerza de corte empleando todas las mediciones aportó evidencia estadística que muestra una clara diferencia significativa ( $\mathrm{p} \leq 0,001)$, entre los promedios de la variable en cada uno de los tratamientos efectuados. El procedimiento de Scheffé posterior comparó el promedio de fuerza de corte para cada uno de los tratamientos experimentales. Los resultados obtenidos se resumen en el Cuadro 5.

En el análisis preliminar de la fuerza de corte, la dispersión de los datos fue grande. Por ello resulta más útil evaluar la fuerza de corte en términos de grupos homogéneos que hacerlo por medio de comparaciones simples y directas entre promedios que a la larga resultan distorsionadas.

Investigaciones anteriores como la realizada por Boleman et al. (1997), estudiaron la aceptación del

Cuadro 5. Grupos homogéneos para los promedios estimados de las fuerzas de corte de cada uno de los tratamientos según la prueba de Scheffé. San José, Costa Rica. 2005.

\begin{tabular}{lccccc}
\hline \multicolumn{1}{c}{$\begin{array}{c}\text { Tratamiento } \\
\text { aplicado }\end{array}$} & $\begin{array}{c}\text { Número } \\
\text { de medi- } \\
\text { ciones }\end{array}$ & \multicolumn{4}{c}{$\begin{array}{c}\text { Grupos homogéneos } \\
(\alpha=\mathbf{0 , 0 5})\end{array}$} \\
& $\mathbf{1}$ & $\mathbf{2}$ & $\mathbf{3}$ & $\mathbf{4}$ \\
\hline Maduración y & & & & & \\
congelación (MC) & 100 & 5,42 & 5,42 & & \\
Maduración (M) & 100 & $\mathbf{5 , 5 1}$ & $\mathbf{5 , 5 1}$ & $\mathbf{5 , 5 1}$ & \\
Maduración con posterior & & & & & \\
cocción y congelación M $\Delta \mathrm{C}$ & 188 & & $\mathbf{5 , 8 8}$ & $\mathbf{5 , 8 8}$ & \\
Maduración y cocción $(\mathrm{M} \Delta)$ & 100 & & & 6,15 & \\
Control & 99 & & & & 7,45 \\
\hline
\end{tabular}

${ }^{1}$ fuerzas de corte en $\mathrm{kg} / \mathrm{cm}^{2}$ consumidor hacia carne con diferentes grados de dureza. Al evaluar tres grupos diferentes el estudio mostró, que aquellas carnes con fuerzas de corte dentro del intervalo $5,90-7,45 \mathrm{~kg} / \mathrm{cm}^{2}$ tenían una aceptación de menos de $1,8 \%$, mientras que aquellas dentro del intervalo 4,08 $-5,40 \mathrm{~kg} / \mathrm{cm}^{2}$ una del 3,6\%. El mayor grado de aceptación era para aquellas carnes entre 2,27-3,58 $\mathrm{kg} / \mathrm{cm}^{2}$, las cuales eran bien recibidas por el $94,6 \%$ de los encuestados.

La tendencia de las fuerzas de corte para el solomo fresco sin ningún tratamiento (control) es hacia un valor promedio de $7,45 \mathrm{~kg} / \mathrm{cm}^{2}$. Este valor es el más alto estimado y es además significativamente diferente de todos los demás grupos de datos. Este hecho permite concluir que cualquiera de los tratamientos aplicados reduce en alguna medida la fuerza de corte inicial del solomo fresco. Se evidencia a la vez como el solomo, previamente a la maduración, es un corte con una dureza que supera el intervalo de $5,90-7,21 \mathrm{~kg} / \mathrm{cm}^{2}$ considerado como de baja aceptación entre los consumidores (Boleman et al. 1997).

Si se estudian los grupos uno, dos y tres, se nota que las fuerzas de corte promedio de varios tratamientos fueron ubicadas estadísticamente dentro de dos o más de estos grupos homogéneos a la vez. Tal es el caso del tratamiento de maduración que es ubicado dentro de los tres grupos. Lo mismo sucede con el tratamiento de maduración seguida de congelación, y ubicado en los grupos uno y dos, y con el tratamiento de maduración, cocción y congelado, que aparece en los grupos dos y tres. Debido a esta particularidad, entre estos grupos intermedios no se pueden establecer diferencias significativas claras que permitan afirmar que las fuerzas de corte promedio de los tratamientos son estadísticamente diferentes entre sí. Al existir valores promedio que son comunes entre todos estos grupos, ninguno de ellos es independiente de los otros, tal y como si sucede con el grupo cuatro correspondiente a la fuerza de corte media del grupo control. Los tratamientos además poseen en términos generales fuerzas de corte que varían entre 5,42 y $6,15 \mathrm{~kg} / \mathrm{cm}^{2}$, valores que si bien son significativamente menores que la fuerza de corte estimada para el solomo fresco, son aún de una dureza alta. Según lo expuesto por Boleman (Boleman et al. 1997), es probable esperar una baja aceptación para estos cortes, aún después de los tratamientos.

Del análisis estadístico se desprenden varios aspectos interesantes. El primero es que a pesar de que los tratamientos presentaron pérdidas de peso desiguales, y especialmente acentuadas en las cocciones, la fuerza de corte promedio final no presenta diferencias significativas. Esto parece confirmar que la jugosidad y contenido general de humedad no están directamente relacionados con la dureza final determinada instrumentalmente. 
La congelación por sí sola, no presenta un efecto sobre la suavidad mayor al logrado con sólo madurar las piezas. Esto puede explicarse en el hecho de que durante los primeros siete días de maduración se dan la gran mayoría de los cambios post mortem asociados con la dureza final (Parrish 1999). Así, al congelar después de la maduración es poco lo que la suavidad puede mejorarse por vía enzimática. Los cambios en cuanto al efecto mecánico que la congelación se presume pueda tener en el tejido conectivo y las proteínas miofibrilares, si es que se registraron, no fueron evidenciados estadísticamente en este estudio. Basándose en lo anterior se puede presumir que la cocción y la congelación, tal y como se plantearon en este estudio, no ofrecieron ningún aporte considerable en la obtención de una mayor suavidad final. Esto plantea la posibilidad de que la mejoría en la suavidad registrada en todos los tratamientos en comparación con las muestras frescas, se deba en su gran mayoría a los cambios acaecidos durante la maduración. Se estaría evidenciando así la estrecha e importante relación existente entre este proceso altamente enzimático y la suavidad final.

Si se consideran aspectos como la simplicidad, el tiempo y el costo de procesamiento, el tratamiento integrado por solo la maduración es el que ofrece mejores resultados. Es recomendable para el solomo el ensayo de métodos alternativos de mejoramiento de la suavidad como puede ser la estimulación eléctrica o la inyección con cloruro de calcio.

\section{LITERATURA CITADA}

AHN, C.; STILES, M. 1990. Antibacterial activity of lactic acid bacteria isolated from vacuum packaged meats. Journal of Applied Bacteriology 69(3): 302-310.

ALAYAN, M. 1997. Calpains and meat tenderness: mode of action and activity regulation (en línea). Consultado 25 ene. 1999. Disponible en: http://www.lincoln.ac.nz/ ansc/moha_www.htm

AMSA (American Meat Science Association). 1978. Guidelines for cookery and sensory evaluation of meat. American Meat Science Association in cooperation with The National Livestock and Meat Board. Chicago. $24 \mathrm{p}$.

A.O.A.C. (Association of Official Analytical Chemistry). 2000. Official methods of analysis. $17^{\text {th }}$ edition. Association of Official Analytical Chemistry. Washington D.C. Chapter 39: 931-932.

BAILEY, A.; RESTALL, D.; SIMS, T.; DUANCE, V. 1979. Meat tenderness. Journal of the Science of Food and Agriculture 30(2): 203-209.
BELITZ, H.W.; GROSCH, W. 1985. Química de los alimentos. Acribia. Zaragoza, España. 813 p.

BOLEMAN, S.J.; BOLEMAN, S.L.; MILLER, R.K.; TAYLOR, J.F.; CROSS, H.R.; WHEELER, T.L.; KOOHMARAIE, M.; SHACKELFORD, S.D.; MILLE, M.F.; WEST, R.L.; JOHNSON, D.D.; SAVELL, J.W. 1997. Consumer evaluation of beef of known categories of tenderness. Journal of Animal Science 75: 1521-1524.

BOSTAN, K.; NAZLI, B.; ARUN, O. O. 2001. Effect of electrical stimulation on meat tenderness and microflora. Veteriner Fakültesi Dergisi 27 (2): 547-556.

BRUCE, H.L.; BALL, R. 1990. Effects of postmortem glycolysis on the quality of hot deboned bovine muscle. Canadian Journal of Animal Science 70(2): 431-439.

BUTS, B.; CLAEYS, E.; DEMEYER, D. 1987. Protein fragmentation and meat tenderness. Mededelingen Van de Faculteit Landbouwwetenschappen Rijksuniversiteit Gent 52 (4): 1529-1538.

CHACÓN, A. 2004. La suavidad de la carne: implicaciones físicas y bioquímicas asociadas al manejo y proceso agroindustrial. Agronomía Mesoamericana 15(2): 225243.

CLAUSEN, I.; LASSEN, A. 2002. Cooking meat - myths, facts and new methods. FodevareRapport 1: 1- 86

COLE, D.J.A; LAWRIE, R.A. 1975. Meat. Ed. AVI. Connecticut, E.E.U.U. 596 p.

COSTELL, E. 1992. Influencia del envase en la aceptabilidad de los alimentos. Revista Española de Ciencia y Tecnología de Alimentos 32(5): 493-507.

DELAZARI, I.; GERALDINI, A.; LEITAO, M.F.; CORTE, O.O. 1980. Incidencia de bacterias esporogénicas anaeróbias em carne bovina. Boletim do Instituto de Tecnologia de Alimentos 17(4): 441-450.

DOBBIE, P.; SINGH, B.; THOMSOM, G.; MERCER, J.; BASS, P.; SPECK, P. 1997. Inter-muscle variation in the calpain system of reed deer: implications for meat tenderness (en línea). Consultado 25 ene. 1999. Disponible en: http://www.nal.usda.govt.nz/www/nzsap/ proc/1995/ab95040.html

DOUMIT, M.; KOOHMARAIE, M. 1999. Inmunoblot analysis of calpastatin degradation: evidence for cleavage by calpain in postmortem muscle (en línea). Consultado 15 ene. 1999. Disponible en: http://www.nal.usda. gov/ttic/tektran/data/

GEESINK, G.; KOOHMARAIE, M. 1998. Effect of calpastatin on degradation of miofibrillar proteins by U-calpain under postmortem conditions (en línea). Consultado 25 ene. 1999. Disponible en: http://www.nal.usda..gov/ttic/ tektran/data/ 
HABER, A.; RUNYON, R. 1973. Estadística general. Editorial Fondo Educativo Interamericano. Estados Unidos de América. 371 p.

HEINEMANN, R. J. B.; PINTO, M. F. 2003. Effect of different concentration of calcium chloride in texture and acceptability of aged beef. Ciência e Tecnologia de Alimentos 23: 146-150

HERRERA, C. 1997. Composición química del tejido muscular de diferentes especies animales. Manual de laboratorio de química de alimentos. Escuela de Química. Universidad de Costa Rica. San Pedro de Montes de Oca. 60 p.

HUERTA-LEIDENZ, N. O.; RODAS-GONZÁLEZ, A. R.; SMITH, G. C. 2004. Effect of vacuum aging and influence of sire on palatability of beef longissimus from grass-fed F1 Senepol x Zebu bulls. Revista Científica, Facultad de Ciencias Veterinarias, Universidad Del Zulia 14(3): 263-269

HWANG, I. H. ; DEVINE, C. E.; HOPKINS, D. L. 2003. The biochemical and physical effects of electrical stimulation on beef and sheep meat tenderness. Meat Science 65 (2): 677-691

JASPER, W.; PLACZEK, R. 1978. Conservación de la carne por el frío. Acribia. Zaragoza. España. 131 p.

JEREMIAH, L.E.; BEAUCHEMIN, K.A.; JONES, S.D.M.; GIBSON, L.L.; RODE, L.M. 1998. The influence of dietary cereal grain source and feed enzymes on the cooking properties and palatability attributes of beef. Canadian Journal of Animal Science 78(3): 271-275.

JOSEPH, R. 1971. Production of tender beef. Food Manufacture 46(10): 29-33.

JUDD, S. 1993. Packaging chilled meat for export. Food Technology in New Zealand 28(8): 32-33.

KOOHMARAIE, M. 1994. Muscle proteinases and meat aging. Meat Science 36 (1/2): 93-104.

KOOHMARAIE, M.; DOUMIT, M.; WHEELER, T. 1996. Meat toughening does not occur when rigor shortening is prevented (en línea). Consultado 25 en. 1999. Disponible en: http://www.nal.usda.gov/ttic/tetran/

KRAMLICH, W.E.; PEARSON, A.M.; TAUBER, F.W. 1975. Processed meats. Avi. Nueva York. USA. 348 p.

MALTIN, C.; BALCERZAK, D; TILLEY, R.; DELDAY, M. 2003. Determinants of meat quality: tenderness. Proceedings of the Nutrition Society 62(2): 337-347

MARCU, A; VINTILA, C. 2000. Variations of $\mathrm{pH}$ of meat in relation to species, age, anatomical part and type of storage. Lucrai Stiintifice Zootehnie si Biotehnologii,
Universitatea de Stiinte Agricole si Medicina Veterinara a Banatului Timisoara 33: 394-397.

MENZIES, D. 1997. Beef industry tries a little tenderness (en línea). Consultado 25 en. 1999. Disponible en: http://www.foodincanada.com/content/1997/03-97/fl1_feactures.html

MERMELSTEIN, N.H. 1993. Controlling E. coli 0157:H7 in meat. Food Technology 47(4): 90-91.

MUELLER, W. 1990. Improvement of the tenderness of roosting meat by mechanical treatment. Mitteilungsblatt der Bundesanstalt fuer Fleischforschung Kulmbach (107): 24-28.

NICOLAI, B.; IMPE, J.; VERLINDEN, B.; MARTENS, T.; VANDEWALLE, J.; BAERDEMAEKER, J. 1993. Predictive modeling of surface growth of lactic acid bacteria in vacuum packaged meat. Food Microbiology 10(3): 229-238.

NISHIMURA, T.; HATTORI, A.; TAKAHASHI, K. 1999. Structural changes in intramuscular connective tissue during the fattening of japanese black cattle: effect of marbling on beef tenderization. Journal of Animal Science 77: 93-104.

OLSON, T. 1998. Can we select for tenderness? (en línea). Consultado 25 ene. 1999. Disponible en: http://gnv.ifas.ufl.edu/ animal/short96/OLSON2.htm

PARRISH, F.C. 1999. Aging of beef (en línea). Consultado 21 ene. 1999. Disponible en: http://www.beef.org/ 8010/beef/librpub/pcrifact/aging.htm

POLIDORI, P.; KAUFFMAN, R.; VALFRE, F. 1996. The effects of electrical stimulation on meat quality. Italian Journal of Food Science 8(3): 183-199.

PRINGLE, T.; WEST, R.; WILLIAMS, S.; JOHNSON, D. 1995. The role of the calpain proteinase system in muscle hypertrophy associated with double muscling in beef (en línea). Consultado 25 ene. 1999. Disponible en: http://www.ads.uga.edu/annrpt/1995/95_061.htm

PRINGLE, T.; WILLIAMS, S.; JOHNSON, D.; WEST, L. 1996. The role of the calpain proteinase system in aged tenderness of Angus and Brahman crossbred steers (en línea). Consultado 8 set. 1998. Disponible en: http://www.ads.uga.edu/annrpt/1996/96_039.htm

QUAGLIA, G.; LOMBARDI, M.; BERTONE, A.; MENESATTI, P. 1992. Effect of enzymatic treatment on tenderness characteristics of freeze-dried meat. Lebensmittel-Wissenschaft und Technologie 25(2): 143-149.

SAVELL, J. 1998. Meat tenderization (en línea). Consultado 15 ene. 1999. Disponible en: http://savel-j.tamu. edu/ tender.html 
SCHEFFÉ, H. 1959. The analysis of variance. Wiley. Londres. $300 \mathrm{p}$.

SCHILLING, M.W; CLAUS, J.R; MARRIOTT, N.G; RAND, A.; SOLOMON, M.B; EIGEL, W.N.; WANG, H. 2002. No effect of hydrodynamic shock wave on protein functionality of beef muscle. Journal of Food Science 67(1): 335-340.

STANTON, C. 1989. Proteolysis induced changes in meat collagen during conditioning. Dissertation Abstracts International 49(12): 5084-5085.

STOUFFER, J. 1975. A method for improving tenderness of carcasses. New York's Food and Life Sciences Quarterly 8(1): 14-18.

TAKAGI, H.; KONDOU, M.; HISATSUKA, T.; NAKAMORI, S.; YING, C.; YAMASAKI, M. 1992. Effects of alkaline elastage from an alkalophilic Bacillus strain on the tenderization of beef meat. Journal of Agriculture and Food Chemistry 40(12): 2364-2368.

TAKAHASHI, K. 1992. Non enzymatic weakening of myofibrillar structures during conditioning of meat: calcium ions at $0.1 \mathrm{mM}$ and their effect on meat tenderization. Biochimie 74(3): 247-250.

TAYLOR, R.; GEESINK, G.H.; THOMPSON, V.F; KOOHMARAIE, M.; GOLL, D.E. 1995. Is Z-disk degradation responsible for postmortem tenderization? Journal of Animal Science 73(5): 1351-1367.

TEXAS A\&M UNIVERSITY. 1999. Meat tenderness (en línea). Consultado 11 ene. 1999. Disponible en: http://www.meat.tamu.edu/tender.html

TROY, D. 1994. Tenderizing beef (en línea). Consultado 19 ene. 1999. Disponible en: http://exp.interspeed.net/ flai/ffe11994.htm

UNIVERSITY OF GUELPH. 1998. Contracting, rigor and conditioning (en línea). Consultado 25 ene. 1999.
Disponible en: http://www.aps.ouguelph.ca/swatland/ $\mathrm{html} / \mathrm{ch} 5.1 . \mathrm{html}$

UNIVERSITY OF ILLINOIS ANIMAL SCIENCES DEPARTMENT. 1999. Water in meat (en línea). Consultado 25 ene. de 1999. Disponible. http://www.ansci.uiuc.edu/meatscience/library/waterinmeat.htm

VANDERZANT, C.; SPITTSTOESSER, D.F. 1992. Compendium of methods for the microbiological examination of food. APHA. Washington. $500 \mathrm{p}$.

VÁZQUEZ, B. I.; CARREIRA, L.; FRANCO, C.; FENTE, C.; CEPEDA, A.; BARROS-VELÁZQUEZ, J. 2004. Shelf life extension of beef retail cuts subjected to an advanced vacuum skin packaging system. European Food Research and Technology 218(2): 118-122

WHEELER, T.; KOOHMARAIE, M.; CROUSE, J. 1992. The effect of postmortem time of injection and freezing on the effectiveness of calcium chloride for improving beef tenderness. Journal of Animal Science (70): 3451-3457.

WHEELER, T.; KOOHMARAIE, M.; SHACKELFORD, S. 1997. Effects of postmortem injection time and postinjection aging time on the calcium activated tenderization process in beef. Journal of Animal Science (75): 2652-2660.

WHIPPLE, G.; KOOHMARAIE, M. 1992. Freezing and calcium chloride marination effects on beef tenderness and calpastatin activity. Journal of Animal Science 70(10): 3081-3085.

WILLIAMS, E.J. 1959. Regression analysis. Wiley. Londres. $300 \mathrm{p}$.

WOODHOUSE, I. 1998. The tender issue of meat (en línea). Consultado 25 ene. 1999. Disponible en: http://wwwaghort.massey.ac.nz/departs/animsc/beef/angus/ a9704.htm 